\title{
Analysis of Kinetic Demixing in a Mixed Oxide $(A, B) O$ in an Oxygen Potential Gradient
}

\author{
I V Belova, M J Brown and G E Murch \\ Diffusion in Solids Group \\ School of Engineering \\ The University of Newcastle \\ Callaghan, NSW 2308 \\ Australia
}

\begin{abstract}
It is well known that homogeneous mixed oxides (A,B)O show kinetic demixing of the cations when exposed at high temperatures to an oxygen potential gradient. This paper shows that when the cation distribution can be described by a random mixing model (the random alloy model) the resulting demixing analysis for the composition profiles can be greatly simplified, and made exact, by making use of the random alloy sum-rule. It is also shown that analyses based on an assumed linearity of the vacancy composition profile will predict incorrect demixing composition profiles for the cations.
\end{abstract}

Keywords: Non-binary oxides; Ionic diffusion; Bulk diffusion; Kinetics; Demixing

\section{Introduction}

When a semi-conducting and initially homogeneous mixed oxide $(\mathrm{A}, \mathrm{B}) \mathrm{O}$ having an essentially immobile oxygen sublattice is exposed to an oxygen potential gradient, the component cations kinetically demix on account of their differing mobilities. The process of kinetic demixing was first studied experimentally in 1979 by Schmalzried et al. [1] in the oxide $(\mathrm{Co}, \mathrm{Mg}) \mathrm{O}$. These workers also provided a sound foundation for the analysis of the demixing process and this foundation has guided all subsequent theoretical work. Since that time, theoretical treatments have either taken a phenomenological approach to describe the composition profiles resulting from the kinetic demixing process [2-4] or an atomistic approach [5-11]. The present paper takes an atomistic approach.

In their original analysis of the steady state composition profiles Schmalzried et al. [1] neglected all off-diagonal phenomenological coefficients. This is, of course, a very common assumption in a preliminary analysis of diffusion problems in solids; see, for example, ref. 12. However, as emphasized by Manning [13], the neglect of off-diagonal phenomenological coefficients may, in some diffusion contexts, be such a poor approximation that the wrong direction of one of the atomic fluxes may be predicted. In their analysis of the steady state composition profiles resulting from kinetic demixing Ishikawa et al. [5] employed the Path Probability Method (PPM) of nonequilibrium statistical mechanics. This particular application of the PPM focussed on the random mixing of the cations on the cation sublattice (the random alloy) model and retained the off-diagonal phenomenological coefficients. However, Ishikawa et al. [5] used a relatively primitive approximation in their analysis for calculating the phenomenological coefficients. Indeed, very substantial differences in the values of the individual phenomenological coefficients predicted from that approximation level have been found when comparing with values obtained from recent high precision Monte Carlo computer simulations [14]. In a later PPM calculation, Wang and Akbar [7] incorporated interactions 
between the cations, but the approximation level was relatively low. Martin [9] specifically explored the effects of including the off-diagonal phenomenological coefficients in the determination of the steady state profiles resulting from kinetic demixing. Martin employed the well-known Manning [15,16] formalism for describing diffusion kinetics in the random alloy with the vacancy mechanism operating. Martin showed that the inclusion of Manning's expressions for the phenomenological coefficients (in terms of the tracer diffusivities) results in a weaker kinetic demixing effect than if it is assumed that the off-diagonal phenomenological coefficient can be neglected. However, a difficulty here is that once again the Manning expressions for the individual phenomenological coefficients (when expressed in terms of the tracer diffusivities) have recently been demonstrated by Monte Carlo computer simulation $[14,17]$ to be not very accurate.

In many areas of diffusion kinetics in solids the random alloy model has proved itself to be extremely useful. Although the random alloy model is usually discussed in the context of composition independent exchange frequencies, an alternative view is that the exchange frequencies can be conceived as average exchange frequencies $[18,19]$. Then the exchange frequencies can be permitted to depend on composition $[18,19]$. In this view, the random mixing of both cations and vacancies is the major underlying assumption in the kinetics formalism and is entirely analogous to the Bragg-Williams approximation of random mixing for the calculation of the chemical potential. This is not a new idea: it has been employed in elementary treatments of diffusion in nonstoichiometric compounds and interstitial solid solutions [20,21].

In the present paper we address the steady state demixing problem using the random alloy model but from a different perspective to what has been done previously. In the present treatment we make direct use of the exact 'sum rule' for diffusion via vacancies in the random alloy model [22]. In Section 2 we show that this leads to a very significant simplification in the whole analysis. We are able to derive exact relations for the gradients of the steady state composition profiles in (A,B)O oxides. In Section 3 it will also be shown that analyses based on an assumed linearity of the vacancy composition profile in the demixing experiment will predict incorrect demixing composition profiles for the cations. In the latter case direct Monte Carlo simulation of demixing will be used to verify the result.

\section{Theory}

We consider a pseudo-binary solid solution of divalent metal oxides e.g. $\mathrm{CoO}$ and $\mathrm{MgO}$ to form $(\mathrm{Co}, \mathrm{Mg}) \mathrm{O}$. The oxygen ions are essentially immobile at the temperatures of interest and so largely act as 'spectators' for the cation diffusion processes. In the usual demixing experimental arrangement one end of the sample is exposed to an oxygen partial pressure (which determines the degree of nonstoichiometry and therefore the cation vacancy composition at this end) whilst the opposite end is exposed to a lower oxygen partial pressure, which again determines the (lower) cation vacancy composition. A composition profile of vacancies is quickly established across the sample by diffusion of both cations. At the first end, some of the oxidisable cations are oxidized to form more oxide at the surface. At the other end, excess oxygen ions evaporate. This results eventually in the sample moving with a steady state velocity in the direction of the higher oxygen partial pressure.

In general, the two types of cation A and B have different mobilities, i.e. they have different exchange rates with the vacancies. Since the two types of cation diffuse in a flux of vacancies the more mobile species tends to move on ahead of the less mobile species. A separation or demixing of the cations occurs across the sample with steady state composition profiles of the cations eventually being reached after long times. Since the vacancy distribution or composition profile is directly linked to that of the cations, the composition profile for the vacancies reaches its final (i.e. steady state) form at the same time as those of the cations. Similarly, the actual vacancy composition at each end of the sample depend on the local 
stoichiometry, which in turn changes with time (because of demixing) until steady state is reached. These latter processes are secondary in importance but need to be taken into account when the time-dependence of the composition profiles is investigated. Our interest here is confined to the steady state condition.

The usual assumption for these oxides is that the electronic mobility is far greater than the atomic mobilities and therefore is not rate determining in the diffusion of the cations. The Onsager flux equations of non-equilibrium thermodynamics for the cation sublattice system can then be written in the well-known way for a binary system with isolated vacancies providing the vehicles for atomic motion $[19,20]$ :

$$
\begin{aligned}
& \mathrm{J}_{\mathrm{A}}=-\mathrm{L}_{\mathrm{AA}} \nabla \mu_{\mathrm{A}}-\mathrm{L}_{\mathrm{AB}} \nabla \mu_{\mathrm{B}}-\mathrm{L}_{\mathrm{Av}} \nabla \mu_{\mathrm{v}} \\
& \mathrm{J}_{\mathrm{B}}=-\mathrm{L}_{\mathrm{AB}} \nabla \mu_{\mathrm{A}}-\mathrm{L}_{\mathrm{BB}} \nabla \mu_{\mathrm{B}}-\mathrm{L}_{\mathrm{Bv}} \nabla \mu_{\mathrm{v}} \\
& \mathrm{J}_{\mathrm{v}}=-\mathrm{L}_{\mathrm{Av}} \nabla \mu_{\mathrm{A}}-\mathrm{L}_{\mathrm{Bv}} \nabla \mu_{\mathrm{B}}-\mathrm{L}_{\mathrm{vv}} \nabla \mu_{\mathrm{v}}
\end{aligned}
$$

where the $L_{i j}$ are the phenomenological coefficients, $\mu_{i}$ is the chemical potential of component $i$ (A, B or V (vacancies)) and $J_{i}$ is the flux of component i. Since $J_{v}=-\left(J_{A}+J_{B}\right)$, then in the usual way, we rewrite Eqns 1 as [23,24]:

$$
\mathrm{J}_{\mathrm{A}}=-\mathrm{L}_{\mathrm{AA}} \nabla\left(\mu_{\mathrm{A}}-\mu_{\mathrm{v}}\right)-\mathrm{L}_{\mathrm{AB}} \nabla\left(\mu_{\mathrm{B}}-\mu_{\mathrm{v}}\right)
$$

and

$$
\mathrm{J}_{\mathrm{B}}=-\mathrm{L}_{\mathrm{AB}} \nabla\left(\mu_{\mathrm{A}}-\mu_{\mathrm{v}}\right)-\mathrm{L}_{\mathrm{BB}} \nabla\left(\mu_{\mathrm{B}}-\mu_{\mathrm{v}}\right)
$$

where we have also made use of the standard reciprocity condition $\mathrm{L}_{\mathrm{AB}}=\mathrm{L}_{\mathrm{BA}}$.

Once steady state demixing of the cations has been achieved, the composition profiles of the cations and vacancies do not change with time. The crystal moves with a steady state velocity, $\mathrm{v}$, with respect to the laboratory frame (which is fixed at an oxygen lattice plane). Schmalzried et al. [1] determined that the condition that leads to steady state is:

$$
\mathrm{J}_{\mathrm{i}}-\mathrm{vc}_{\mathrm{i}}=0 \quad \mathrm{i}=\mathrm{A}, \mathrm{B}
$$

where $c_{i}$ is the composition of component $i$ (with respect to the cation sublattice).

Eqns 2 and 3 then lead to the usual result [5-7]:

$$
\begin{aligned}
& \nabla\left(\mu_{\mathrm{A}}-\mu_{\mathrm{v}}\right)=-\frac{\left(\mathrm{L}_{\mathrm{BB}} \mathrm{c}_{\mathrm{A}}-\mathrm{L}_{\mathrm{AB}} \mathrm{c}_{\mathrm{B}}\right) \mathrm{v}}{\mathrm{L}_{\mathrm{AA}} \mathrm{L}_{\mathrm{BB}}-\mathrm{L}_{\mathrm{AB}}{ }^{2}} \\
& \nabla\left(\mu_{\mathrm{B}}-\mu_{\mathrm{v}}\right)=-\frac{\left(\mathrm{L}_{\mathrm{AA}} \mathrm{c}_{\mathrm{B}}-\mathrm{L}_{\mathrm{AB}} \mathrm{c}_{\mathrm{A}}\right) \mathrm{v}}{\mathrm{L}_{\mathrm{AA}} \mathrm{L}_{\mathrm{BB}}-\mathrm{L}_{\mathrm{AB}}{ }^{2}}
\end{aligned}
$$

Eqns 4 gives the ratio:

$$
\frac{\nabla\left(\mu_{\mathrm{A}}-\mu_{\mathrm{v}}\right)}{\nabla\left(\mu_{\mathrm{B}}-\mu_{\mathrm{v}}\right)}=\frac{\mathrm{L}_{\mathrm{BB}} \mathrm{c}_{\mathrm{A}}-\mathrm{L}_{\mathrm{AB}} \mathrm{c}_{\mathrm{B}}}{\mathrm{L}_{\mathrm{AA}} \mathrm{c}_{\mathrm{B}}-\mathrm{L}_{\mathrm{AB}} \mathrm{c}_{\mathrm{A}}}
$$


Eqns 4 and 5 are often referred to as the 'demixing equations'. They provide the basic relationships between the thermodynamic driving forces and the phenomenological coefficients relevant to the demixing experiment.

At this point, we now introduce the exact sum-rule for the random alloy [22]:

$$
\begin{aligned}
& \mathrm{L}_{\mathrm{AA}}=\mathrm{c}_{\mathrm{v}} \mathrm{c}_{\mathrm{A}} \mathrm{w}_{\mathrm{A}} \mathrm{K}-\frac{\mathrm{w}_{\mathrm{A}}}{\mathrm{w}_{\mathrm{B}}} \mathrm{L}_{\mathrm{AB}} \\
& \mathrm{L}_{\mathrm{BB}}=\mathrm{c}_{\mathrm{v}} \mathrm{c}_{\mathrm{B}} \mathrm{w}_{\mathrm{B}} \mathrm{K}-\frac{\mathrm{w}_{\mathrm{B}}}{\mathrm{w}_{\mathrm{A}}} \mathrm{L}_{\mathrm{AB}}
\end{aligned}
$$

where $\mathrm{K}=\mathrm{Na}^{2} \mathrm{kT}, \mathrm{N}$ is the number of sites per unit volume, $\mathrm{a}$ is the lattice parameter, and $\mathrm{k}$ and $\mathrm{T}$ have their usual meanings, and $\mathrm{w}_{\mathrm{A}}$ and $\mathrm{w}_{\mathrm{B}}$ are the exchange rates of $\mathrm{A}$ and $\mathrm{B}$ cations with a vacancy. The first term on each of the right hand sides of Eqns 6 are the uncorrelated parts, $\mathrm{L}_{\mathrm{AA}}{ }^{(0)}$ and $\mathrm{L}_{\mathrm{BB}}{ }^{(0)}$, of $\mathrm{L}_{\mathrm{AA}}$ and $\mathrm{L}_{\mathrm{BB}}$ respectively $[23,24]$. For the binary alloy the existence of the sum-rule means that there is only one independent phenomenological coefficient. Eqn 6 is also exact for any site fraction of vacancies.

On substitution of Eqns 6 into Eqns 4 we soon have that

$$
\begin{aligned}
& \nabla\left(\mu_{\mathrm{A}}-\mu_{\mathrm{v}}\right)=-\frac{\mathrm{v}}{\mathrm{c}_{\mathrm{v}} \mathrm{Kw}_{\mathrm{A}}}=-\frac{\mathrm{vc}_{\mathrm{A}}}{\mathrm{L}_{\mathrm{AA}}^{(0)}} \\
& \nabla\left(\mu_{\mathrm{B}}-\mu_{\mathrm{v}}\right)=-\frac{\mathrm{v}}{\mathrm{c}_{\mathrm{v}} \mathrm{Kw}_{\mathrm{B}}}=-\frac{\mathrm{vc}_{\mathrm{B}}}{\mathrm{L}_{\mathrm{BB}}^{(0)}}
\end{aligned}
$$

and Eqn 5 then simply becomes:

$$
\frac{\nabla\left(\mu_{\mathrm{A}}-\mu_{\mathrm{v}}\right)}{\nabla\left(\mu_{\mathrm{B}}-\mu_{\mathrm{v}}\right)}=\frac{\mathrm{w}_{\mathrm{B}}}{\mathrm{w}_{\mathrm{A}}}
$$

Eqns 7 and 8 are exact for the random alloy model. Note the surprising absence of off-diagonal terms in these equations. Recently, the authors [22] showed, as a result of the random alloy sum-rule, that the ratio of the intrinsic diffusivities in the random alloy is given by:

$$
\frac{\mathrm{D}_{\mathrm{A}}^{\mathrm{I}}}{\mathrm{D}_{\mathrm{A}}^{\mathrm{I}}}=\frac{\mathrm{w}_{\mathrm{A}}}{\mathrm{w}_{\mathrm{B}}}
$$

In the context of mixed oxides, these intrinsic diffusivities would be those pertaining to the cations in an interdiffusion experiment, say involving a couple AO/BO. Eqn 8 then gives the interesting result (exact for the random alloy) for the demixing situation:

$$
\frac{\nabla\left(\mu_{\mathrm{A}}-\mu_{\mathrm{v}}\right)}{\nabla\left(\mu_{\mathrm{B}}-\mu_{\mathrm{v}}\right)}=\frac{\mathrm{D}_{\mathrm{B}}^{\mathrm{I}}}{\mathrm{D}_{\mathrm{A}}^{\mathrm{I}}}
$$

In order to proceed, we convert the chemical potential gradients to composition gradients $[5,6,23,24]$ :

$$
\nabla\left(\mu_{i}-\mu_{v}\right)=\frac{\partial\left(\mu_{i}-\mu_{v}\right)}{\partial c_{A}} \nabla c_{A}+\frac{\partial\left(\mu_{i}-\mu_{v}\right)}{\partial c_{B}} \nabla c_{B} \quad i=A, B
$$

This soon leads to the following coupled set of ordinary differential equations describing the steady state composition profiles: 


$$
\begin{aligned}
\frac{d c_{A}}{d \xi} & =\frac{v}{c_{v} K \Delta_{\mu}}\left(-\frac{m_{22}}{w_{A}}+\frac{m_{12}}{w_{B}}\right) \\
\frac{d c_{B}}{d \xi} & =\frac{v}{c_{v} K \Delta_{\mu}}\left(\frac{m_{21}}{w_{A}}-\frac{m_{11}}{w_{B}}\right) \\
\frac{d c_{v}}{d \xi}=-\frac{d c_{A}}{d \xi}-\frac{d c_{B}}{d \xi} & =\frac{v}{c_{v} K \Delta_{\mu}}\left(\frac{m_{22}-m_{21}}{w_{A}}+\frac{m_{11}-m_{12}}{w_{B}}\right)
\end{aligned}
$$

where $\xi$ represents a coordinate along the length of the moving sample and $m_{i j}=\partial\left(\mu_{i}-\mu_{v}\right) / \partial c_{j}$, where $\mathrm{i}, \mathrm{j}=\mathrm{A}, \mathrm{B}$, and $\Delta_{\mu}=\mathrm{m}_{11} \mathrm{~m}_{22}-\mathrm{m}_{12} \mathrm{~m}_{21}$.

If we now assume thermodynamic ideality, which is strictly appropriate for the random alloy model, then we can write that $\mu_{\mathrm{i}}-\mu_{\mathrm{v}}=\ln \left(\mathrm{c}_{\mathrm{i}} / \mathrm{c}_{\mathrm{v}}\right)$. Then Eqn 12 can be reduced to:

$$
\begin{aligned}
\frac{\mathrm{dc}_{\mathrm{A}}}{\mathrm{d} \xi} & =\frac{\mathrm{c}_{\mathrm{A}} \mathrm{v}}{\mathrm{c}_{\mathrm{v}} \mathrm{K}}\left(\frac{\mathrm{c}_{\mathrm{A}}-1}{\mathrm{w}_{\mathrm{A}}}+\frac{\mathrm{c}_{\mathrm{B}}}{\mathrm{w}_{\mathrm{B}}}\right) \\
\frac{\mathrm{dc}_{\mathrm{B}}}{\mathrm{d} \xi} & =\frac{\mathrm{c}_{\mathrm{B}} \mathrm{v}}{\mathrm{c}_{\mathrm{v}} \mathrm{K}}\left(\frac{\mathrm{c}_{\mathrm{A}}}{\mathrm{w}_{\mathrm{A}}}+\frac{\mathrm{c}_{\mathrm{B}}-1}{\mathrm{w}_{\mathrm{B}}}\right) \\
\frac{\mathrm{dc}_{\mathrm{v}}}{\mathrm{d} \xi} & =\frac{\mathrm{v}}{\mathrm{K}}\left(\frac{\mathrm{c}_{\mathrm{A}}}{\mathrm{w}_{\mathrm{A}}}+\frac{\mathrm{c}_{\mathrm{B}}}{\mathrm{w}_{\mathrm{B}}}\right)
\end{aligned}
$$

Eqns 13 have been obtained previously by Ishikawa et al. [5] using approximate expressions obtained from the PPM for the $\mathrm{L}_{\mathrm{ij}}$ in the random alloy with vanishingly small vacancy content. Incidentally, in that study $\mathrm{K}=1$ because of the scale chosen for the flux equations. Eqns 13 as derived here are exact for the random alloy model and for any value of the vacancy composition. Its form is purely a consequence of the sum-rule and, since approximate diffusion kinetics treatments based on the random alloy model should, in principle, satisfy the sum-rule, then, inevitably, all such treatments will give Eqn 13.

Focussing on the third equation in Eqns 13, we can integrate it directly (when $\mathrm{w}_{\mathrm{A}}, \mathrm{w}_{\mathrm{B}}$ are independent of $\xi$ ) to give:

$$
\mathrm{c}_{\mathrm{v}}^{2}-\mathrm{c}_{\mathrm{v}}^{1}=\frac{\mathrm{v}}{\mathrm{K}}\left(\frac{\ell}{\mathrm{w}_{\mathrm{A}}} \mathrm{c}_{\mathrm{A}}^{0}+\frac{\ell}{\mathrm{w}_{\mathrm{B}}} \mathrm{c}_{\mathrm{B}}^{0}\right)
$$

which then provides the following expression for $\mathrm{v}$ :

$$
\mathrm{v}=\left(\mathrm{c}_{\mathrm{v}}^{2}-\mathrm{c}_{\mathrm{v}}^{1}\right) \mathrm{K}\left(\frac{\ell}{\mathrm{w}_{\mathrm{A}}} \mathrm{c}_{\mathrm{A}}^{0}+\frac{\ell}{\mathrm{w}_{\mathrm{B}}} \mathrm{c}_{\mathrm{B}}^{0}\right)^{-1}
$$

where $\ell$ is the length of the specimen under the steady-state conditions, $\mathrm{c}_{\mathrm{A}}{ }^{0}$ and $\mathrm{c}_{\mathrm{B}}{ }^{0}$ are the average atomic compositions in the sample, $\mathrm{c}_{\mathrm{v}}{ }^{2}$ is the (steady state) vacancy composition at the higher oxygen partial pressure end and $\mathrm{c}_{\mathrm{v}}{ }^{1}$ is the vacancy composition at the opposite (lower oxygen partial pressure) end.

With the expression Eqn 14 in hand, we can now rewrite Eqns 13 in the following way:

$$
\frac{\mathrm{dc}_{\mathrm{A}}}{\mathrm{d} \xi^{\prime}}=\frac{\Delta_{\mathrm{v}} \mathrm{c}_{\mathrm{A}}\left[\left(\mathrm{c}_{\mathrm{A}}-1\right) \overline{\mathrm{w}}+\mathrm{c}_{\mathrm{B}}\right]}{\mathrm{c}_{\mathrm{v}}\left[\mathrm{c}_{\mathrm{A}}^{0} \overline{\mathrm{w}}+\mathrm{c}_{\mathrm{B}}^{0}\right]}
$$




$$
\begin{aligned}
\frac{\mathrm{dc}_{\mathrm{B}}}{\mathrm{d} \xi^{\prime}} & =\frac{\Delta_{\mathrm{v}} \mathrm{c}_{\mathrm{B}}\left[\mathrm{c}_{\mathrm{A}} \overline{\mathrm{w}}+\mathrm{c}_{\mathrm{B}}-1\right]}{\mathrm{c}_{\mathrm{v}}\left[\mathrm{c}_{\mathrm{A}}^{0} \overline{\mathrm{w}}+\mathrm{c}_{\mathrm{B}}^{0}\right]} \\
\frac{\mathrm{dc}_{\mathrm{v}}}{\mathrm{d} \xi^{\prime}} & =\Delta_{\mathrm{v}} \frac{\mathrm{c}_{\mathrm{A}} \overline{\mathrm{w}}+\mathrm{c}_{\mathrm{B}}}{\mathrm{c}_{\mathrm{A}}^{0} \overline{\mathrm{w}}+\mathrm{c}_{\mathrm{B}}^{0}}
\end{aligned}
$$

where we have defined $\xi^{\prime}=\xi / \ell, \overline{\mathrm{w}}=\mathrm{w}_{\mathrm{B}} / \mathrm{w}_{\mathrm{A}}$ and $\Delta_{\mathrm{v}}=\mathrm{c}_{\mathrm{v}}{ }^{2}-\mathrm{c}_{\mathrm{v}}{ }^{1}$.

It should be especially noted that in Eqns 15 all quantities are in a dimensionless form. Accordingly, Eqns 13 can thus be used to justify that atomistic type calculations of the demixing profiles in small simulation systems [8] are indeed applicable to the analysis of the corresponding experimental data in macroscopic systems.

\section{Discussion}

Ishikawa et al. [5] were able to provide an analytical solution to the coupled ordinary differential equations (Eqns 13) by assuming a linear steady state vacancy composition profile across the sample. However, the form of $\mathrm{dc}_{\mathrm{v}} / \mathrm{d} \xi$ in Eqn 15 would suggest that this assumption is unlikely to be a good one unless $\overline{\mathrm{w}} \approx 1$ and $\mathrm{c}_{\mathrm{v}} \rightarrow 0$. In the present case we solved Eqns 15 (the second and third) using standard numerical methods for $\overline{\mathrm{w}}=1.0,0.25$ and 0.1 . Results are shown in Figs $1 \mathrm{a}, \mathrm{b}$ as solid lines for $\overline{\mathrm{w}}=0.1$ and 0.25 for the steady state demixed composition profiles of the cations and Fig 2 for $\overline{\mathrm{W}}=0.1,0.25$ and 1.0 for the steady state composition profiles of the vacancies. Also included in these figures are corresponding Monte Carlo computer simulation results using the same Monte Carlo computer simulation program employed by Zhang et al. [8] to generate steady state demixed cation composition profiles using the random alloy model. It can be seen that the simulations and the numerical solution agree extremely well with each other, as of course they should. The non-linearity of the vacancy composition profiles in Fig 2 should be especially noted when $\overline{\mathrm{w}} \neq 1.0$. It is then naturally of interest to investigate the effect on the cation composition profiles of the assumption of a linear vacancy composition profile as assumed in [5]. These are shown in Fig $1 \mathrm{a}, \mathrm{b}$ as dashed lines. The cation composition profiles differ very significantly from the correct ones indicating that this is a rather poor assumption. It is clearly far better to solve the coupled ordinary differential equations numerically than to employ the approximate analytical solution.

From a practical point of view, if one has in-hand the steady state cation composition profiles that have resulted from a known vacancy composition gradient across the mixed oxide sample, then they can be compared with the numerical solutions until a good match is found. Such matching will give the ratio of the cation exchange frequencies $\mathrm{w}_{\mathrm{B}} / \mathrm{w}_{\mathrm{A}}$. The implication in doing this is that the exchange frequency ratio is independent of composition. However, in principle, a composition dependence $[18,19]$ can be allowed for in the numerical solutions. If the intrinsic diffusivities of the cations happen to be available as a function of composition then their ratio can be compared directly with the exchange frequency ratio (Eqn 9). On the other hand, if tracer diffusivities of the cations are available then these need to be processed to extract $\mathrm{w}_{\mathrm{B}} / \mathrm{w}_{\mathrm{A}}$. Traditionally, this is usually achieved using the Manning diffusion kinetics formalism for the random alloy $[15,16]$. Recently, it has become clear from high precision Monte Carlo simulations that the Manning formalism is not particularly accurate for this task [17]. A far better formalism for the purposes of processing the tracer diffusivities is the selfconsistent formalism of Moleko et al. [26], which has been shown to be almost exact [17]. For details of the method of processing the tracer diffusivities, see [17].

\section{Conclusions}

In this paper we have shown that when the cation distribution can be described by a random mixing model (the random alloy model), the resulting theoretical analysis for the 
steady state demixing composition profiles can be greatly simplified, and made exact, by making use of the random alloy sum-rule. It has also been shown that analyses based on an assumed linearity of the vacancy composition profile would predict incorrect steady state demixing composition profiles for the cations with the deviations becoming larger as the difference in the exchange frequencies increases.

Acknowledgments

We wish to thank the Australian Research Council (Large Grants and Discovery Project Grants Schemes) for its support of this research. One of us (IVB) also wishes to thank the Australian Research Council for the award of a Queen Elizabeth II Fellowship. We wish to thank Dr Ling Zhang (C.S.I.R.O.) for her assistance with the computer simulation.

\section{REFERENCES}

[1] Schmalzried H, Laqua W and Lin PL. Z. Naturf. 1979;34a:192.

[2] Vedula K. Oxid. Met. 1987;28: 99.

[3] Hong J-O, Teller O, Martin M, Yoo H-I. Sol. St. Ionics 1999;123:75.

[4] Monceau D, Petot C and Petot-Ervas G. Solid State Ionics 1999;45:231.

[5] Ishikawa T, Sato, H, Kikuchi R, and Akbar SA. J. Am. Ceram. Soc. 1985;68:1.

[6] Ishikawa T, Akbar SA Zhu W and Sato H. J. Am. Ceram. Soc. 1988;71:513.

[7] Wang CC and Akbar SA. J Phys D. Appl Phys. 1995;28:120.

[8] Zhang L and Murch GE. Phil. Mag A 1990;62:267.

[9] Martin M. Ceram. Trans. 1991;24:91.

[10] Martin M and Schmackpfeffer R. Ber. Bunsenges. Phys. Chem. 1989;93:1271.

[11] Martin M and Schmackpfeffer R. Sol. St. Ionics 1994;72:67.

[12] Kirkaldy JS, Young DJ. Diffusion in the condensed state. London: Insitute of metals, 1987.

[13] Manning JR. Metal. Trans. 1970;504:499.

[14] Belova IV and Murch GE. Phil Mag A 2000;80:599.

[15] Manning JR. Diffusion kinetics for atoms in crystals. Princeton: Van Nostrand, 1968.

[16] Manning JR. Phys. Rev. B 1971;4:1111.

[17] Belova IV and Murch GE. Phil Mag A 2000;80:1469.

[18] Belova IV and Murch GE. Def Diffus Forum 2002;203-205:125.

[19] Belova IV and Murch GE. Mass and charge transport in inorganic materials. Faenza: Techna, accepted for publication and in press.

[20] Murch GE. Acta Met. 1979;27:1701.

[21] Murch GE. J Phys Chem Solids 1981;42:227.

[22] Moleko LK, Allnatt AR and Allnatt EL. Phil Mag A 1989;59:141.

[23] Howard RE and Lidiard AB. Rep. Prog. Phys. 1964;27:161.

[24] Allnatt AR and Lidiard AB. Atomic transport in solids. Cambridge: University Press, 1993.

[25] Belova IV and Murch GE. Phil Mag Lett 2001;81:661.

[26] Moleko LK, Allnatt AR and Allnatt EL, Phil Mag A 1989;59:141. 
Figure Captions

Figure 1. The steady-state cation composition profiles for the cases $\overline{\mathrm{w}}=\mathrm{w}_{\mathrm{B}} / \mathrm{w}_{\mathrm{A}}=0.1$ (a) and $\overline{\mathrm{W}}=\mathrm{w}_{\mathrm{B}} / \mathrm{w}_{\mathrm{A}}=0.25$ (b). Symbols: Monte Carlo simulation results, solid lines: numerical solution of Eqns 15 (second and third equations), dashed lines: approximate solution [5] to Eqns 15.

Figure 2. The steady-state vacancy composition profiles for the cases $\overline{\mathrm{w}}=\mathrm{w}_{\mathrm{B}} / \mathrm{w}_{\mathrm{A}}=0.1,0.25$ and 1.0. Monte Carlo simulation results o: $\mathrm{w}_{\mathrm{B}} / \mathrm{w}_{\mathrm{A}}=1.0, \Delta: \mathrm{w}_{\mathrm{B}} / \mathrm{w}_{\mathrm{A}}=0.25, \square: \mathrm{w}_{\mathrm{B}} / \mathrm{w}_{\mathrm{A}}=0.1$, solid lines: numerical solution of Eqns 15 (second and third equations). 